\title{
Study of Rice Processing System Life Cycle
}

\section{Liting Ge, Qingxin Fan*, Yang Song}

School of Municipal and Environmental Engineering, Harbin Institute of Technology, Harbin, China

\section{Email address:}

gelt_hit@163.com (Liting Ge), fanqingxin@hit.edu.cn (Qingxin Fan), 604826641@qq.com (Yang Song)

${ }^{*}$ Corresponding author

\section{To cite this article:}

Liting Ge, Qingxin Fan, Yang Song. Study of Rice Processing System Life Cycle. Science Discovery. Vol. 5, No. 4, 2017 , pp. $276-282$. doi: 10.11648/j.sd.20170504.16

Received: April 30, 2017; Accepted: June 1, 2017; Published: June 6, 2017

\begin{abstract}
This study attempts to evaluate the environmental impact of rice processing system (raw materials acquisition, paddy processing, packaging, transportation and by-product recycle) with the application of GaBi6.0 software and CML2001-Jan.2016 model, primary data were collected from an agricultural group in the northeast China. The results that the life cycle total environmental impact is $4.35 \times 10-6$, and Global Warming Potential causes the largest damage on environment with the contribution of $66.58 \%$ of the total environmental impact potential. Followed by Freshwater Aquatic Ecotoxicity Potential, with the contribution proportion of $18.68 \%$. The rice planting stage is the main steps of an impact on the environment, with the contribution proportion of $88.4 \%$. Improving the way of irrigation, regulating fertilizers and pesticides can diminish the environmental impact of the whole system.
\end{abstract}

Keywords: Rice, Life Cycle Assessment, GaBi6.0 Software, Environmental Impact

\section{大米加工系统的生命周期的研究}

\section{葛立婷, 樊庆锌 ${ }^{*}$, 宋洋}

市政环境工程学院, 哈尔滨工业大学, 哈尔滨, 中国

邮箱

gelt_hit@163.com（葛立婷）, fanqingxin@hit.edu.cn（樊庆锌）, 604826641@qq.com（宋洋）

摘要: 本文以东北某农业集团为例, 利用GaBi6.0软件, 选用CML2001-Jan.2016模型对大米加工系统的全生命周期进行 环境影响分析, 系统范围涵盖原料获取、稻谷加工及包装、运输和副产品再利用。结果表明, 大米加工系统总的环境 影响为 $4.35 \times 10^{-6}$, 全球变暖是系统对环境影响的主要类型, 占总环境影响的 $66.58 \%$; 其次是淡水生态毒性, 占总环境 影响的 $18.68 \%$ 。稻谷种植阶段是对环境产生影响的主要环节, 占总环境影响的 $88.4 \%$, 改进水稻灌溉方式（如地下滴 灌），减少化肥和农药的用量，可有效降低系统环境影响。

关键词: 大米, 生命周期评价, GaBi6.0, 环境影响

\section{1. 引言}

大米、小麦和玉米是世界三大粮食作物, 其中大米产 量占世界谷物总产量的 $1 / 3$ 左右 [1], 有超过一半的人口将 大米作为获取卡路里和蛋白质的主要来源。据统计, 近几
年中国稻米产量连年增加[2], 庞大的稻米生产及市场需求 在给我们带来经济效益的同时也对资源环境产生较大的 影响。科学地分析大米全生命周期各阶段的资源消耗和污 染排放, 能够为制定污染减排措施提供有力支持。

生命周期评价通过量化产品系统的物资投入和产出 评估系统全生命周期对环境产生的潜在影响。利用生命周 
期评价理论对产品进行分析能够更直观快捷地找出能耗 高、物耗高、污染重的部位及环节[3]。目前, 很多国家对 大米的环境影响分析在种植、加工、运输、食用阶段均有 涉及, 如Kwofie等[4]利用生命周期评价对比分析了农村半 煮熟和集成蒸汽烘干两种稻谷加工方式; Roy等 [5]针对大 米的不同消费模式进行了生命周期分析; Frischknecht等 [6] 分析了中国、印度、美国一瑞士三个不同地区农业生产、 稻谷加工、配送和烹饪过程的生命周期清单数据。而中国 对大米的环境影响分析仅停留在大米种植阶段, 本文拟通 过GaBi6.0软件建模对大米加工系统全生命周期进行分析, 并对其生命周期中的关键影响因子做敏感性分析。

\section{2. 研究对象概况}

由于中国大米加工行业企业加工规模、加工技术和装 备水平、副产品综合利用程度参差不齐, 对整个大米加工 行业的环境影响分析较为困难。同时, 考虑到近几年中大 型稻谷加工企业迅速增加, 行业发展也越来越趋于品牌化、 规模化、产业化[7], 本文选取年加工稻谷 30 万吨的东北某 农业集团作为案例进行分析。

该农业集团年产精品大米约19万吨, 其稻谷加工工艺 为初清烘干 $\rightarrow$ 稻谷清选 $\rightarrow$ 聋谷 $\rightarrow$ 厚度分级 $\rightarrow$ 碾米 (3-4次) $\rightarrow$ 分级 $\rightarrow$ 配米 $\rightarrow$ 色选 $\rightarrow$ 包装。副产品稻壳代替煤炭用于稻 谷干燥和发电, 燃烧稻壳产生的电量部分供本企业使用, 其余大部分进入电网; 碎米经磨粉机研磨 $\rightarrow$ 篮理制取米粉; 米糠经膨化造粒 $\rightarrow$ 浸出 $\rightarrow$ 混油处理 $\rightarrow$ 油脂精炼 (脱胶、脱 酸、脱色脱臭、脱蜡等) 制取米糠油。

\section{3. 大米加工系统的生命周期评价}

\section{1. 目的和范围的确定}

本文基于东北地区某农业集团的实际情况，对大米 加工系统进行生命周期分析, 以期发现对环境产生影响 的重要环节, 为企业及同行业清洁生产决策的制定提供 参考。

本次研究系统边界定义为“从稻谷进厂到副产品再利 用”全过程, 包括原材料获取 (稻谷和塑料编织袋) 、稻 谷烘干、稻谷加工、包装、运输、副产品利用 (稻壳发电、 碎米制米粉、米糠制米糠油)，系统内不考虑消费者消费、 基础设施建设和企业员工生活带来的能源资源消耗和污 染排放, 具体系统边界见图1。其中, 环保单元主要为: 稻谷加工过程中处理车间粉尘的脉冲除尘器和处理烘干 塔烟尘的干湿两级除尘器; 稻壳发电过程中的烟气处理设 施（SNCR脱硝+炉内喷钙脱硫+布袋除尘器）。功能单元 设定为企业大米年产量19万吨。

\section{2. 清单分析}

\subsection{1. 数据收集}

本次研究中大米生产加工过程和稻壳发电过程的相 关数据通过对东北地区某农业集团实地调研获得, 其他环 节的数据通过PE和Ecoinvent数据库或相关文献资料获得。

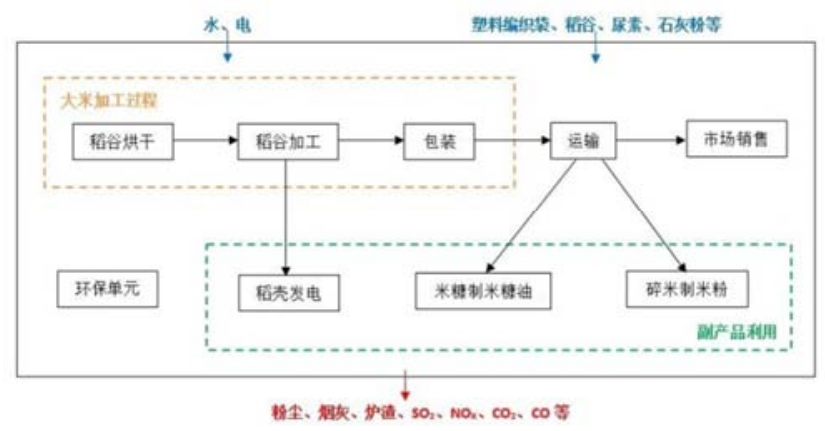

图1 系统边界图。

\section{1）大米加工}

原材料稻谷和水获取过程的资源消耗和污染排放数 据来源于 GaBi6.0软件数据库; 烘干燃料为企业副产品稻 壳, 消耗的电量由企业稻壳发电系统供应, 这部分数据通 过实地调研获得; 包装材料塑料编织袋有 $10 \mathrm{~kg} 、 25 \mathrm{~kg}$ 和 $50 \mathrm{~kg}$ 三种规格, 单位面积质量按 $50 \mathrm{~g}$ 计。大米加工过程的 输入输出数据见表 1 。

表1 大米加工过程输入输出数据统计表。

\begin{tabular}{lll}
\hline 名称 & $\begin{array}{l}\text { 年消耗/年产 } \\
(\text { 年排放 })\end{array}$ & 备注 \\
\hline 电 & $\begin{array}{l}9.5 \times 10^{6} \mathrm{kwh} \\
\text { 水 }\end{array}$ & 厂内稻壳发电供电 \\
稻谷 & $5700 \mathrm{~m}^{3}$ & 市政管网自来水 \\
& $300000 \mathrm{t}$ & 含水 $\leq 17 \%$ \\
塑料编织袋 & 100 万个 & 容量 $10 \mathrm{~kg} /$ 袋, $38 \times 58 \times 1$ \\
& 720 万个 & 容量 $25 \mathrm{~kg} /$ 袋, $44 \times 77 \times 1$ \\
中包装精制大米 & $10000 \mathrm{t}$ & 容量 $50 \mathrm{~kg} /$ 袋, $95 \times 55 \times 1$ \\
大包装精制大米 & $180000 \mathrm{t}$ & 外售 \\
米糠 & $30900 \mathrm{t}$ & 外售 \\
稻壳 & $52500 \mathrm{t}$ & 外售 \\
稻壳灰、捕集粉尘 & $244.77 \mathrm{t}$ & 烘干塔燃烧和发电 \\
碎米粒及异色粒 & $6000 \mathrm{t}$ & 外售 \\
粉尘 & $8.852 \mathrm{t}$ & 外售 \\
稻草等杂物 & $14346 \mathrm{t}$ & 大米加工车间 \\
$\mathrm{SO}_{2}$ & $2.52 \mathrm{t}$ & 制米车间 \\
$\mathrm{NO}_{\mathrm{x}}$ & $2.52 \mathrm{t}$ & 烘干塔热风炉烟气 \\
烟尘 & $3.168 \mathrm{t}$ & 烘干塔热风炉烟气 \\
$\mathrm{CO}_{2}$ & $2030.6 \mathrm{t}$ & 烘干塔热风炉烟气 \\
\hline
\end{tabular}

\section{2) 副产品利用}

稻壳发电工程年消耗稻壳 $5.1 \times 10^{4} \mathrm{t}$, 发电 $48.8 \times 10^{6} \mathrm{kWh}$, 用电 $3.9 \times 10^{5} \mathrm{kWh}$ 。年耗水 $1.63 \times 10^{5} \mathrm{t}$ 、脱硫剂石灰粉 $56 \mathrm{t}$ 、 脱硝剂尿素 $13 \mathrm{t}$ 、锅炉点火助燃油轻柴油 (夏季采用 0 号柴 油, 冬季采用-20号柴油） $1.2 \mathrm{t}$, 其获取过程的资源消耗和 污染排放数据来源于 $\mathrm{GaBi6}$.0软件数据库。发电过程中年 排放 $\mathrm{SO}_{2}$ 约 $19.58 \mathrm{t}, \mathrm{NO}_{\mathrm{X}}$ 约 $48.17 \mathrm{t}$, 颗粒物约 $11.52 \mathrm{t}, \mathrm{CO}_{2}$ 约 $69040.4 \mathrm{t}$, 污水处理泥沙约 $0.2 \mathrm{t}$, 燃烧灰渣约 $6619 \mathrm{t}$ 。烟气 中 $\mathrm{PM}_{10}$ 和 $\mathrm{PM}_{2.5}$ 的排放系数参考相关文献 $[8]$ 。

米糠制油环节输入的原料和能源包括米糠、正己烷、 磷酸、盐、柠檬酸、烧碱、白土、稻壳、电、水等, 输出 的污染主要是颗粒物、 $\mathrm{SO}_{2} 、 \mathrm{NO}_{\mathrm{X}} 、 \mathrm{COD}$ 、氨氮、废油、 滤渣等, 具体数据参考佳木斯市通四海稻米油深加工有限 公司日加工150t米糠制油项目环境影响报告书, 柠檬酸、 白土等部分原材料制造阶段的资源消耗和污染排放数据 
获取困难, 本次研究不予考虑。碎米制米粉过程主要消耗 电能, 用电量参考相关文献[9]。

\section{3) 运输}

根据系统边界的划定, 本次研究不考虑稻谷、辅助材 料及包装材料运输到厂过程。根据企业实际情况, 大米和
米糠需长途运输, 采用铁路、公路结合的运输方式; 碎米 粒、稻壳灰和捕集粉尘运输距离较短, 只采取公路运输。

\subsection{2. 软件建模}

根据上述清单数据, 运用 $\mathrm{GaBi}$.0.0软件建立大米加工 系统的生命周期模型, 如图2所示。

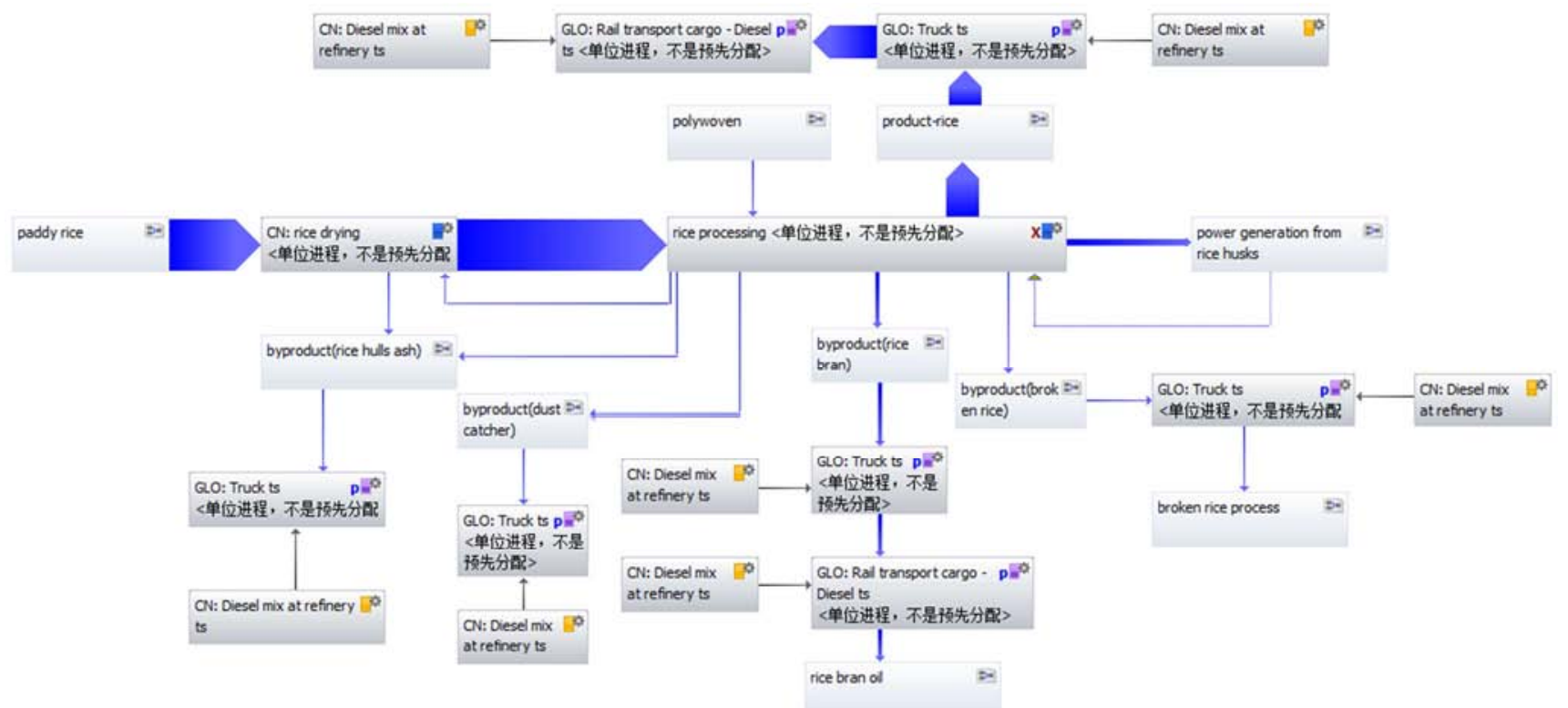

图2 软件模型示意图。

\subsection{3. 物流、能流和排放的分配}

大米加工系统产出大米、稻壳、米糠和碎米多种产品, 并将稻壳、米糠和碎米再利用，因而需要考虑产品和各子 系统物流、能流和排放的分配问题。在大米加工过程中， 选取产品质量作为分配参数, 以每种产品的相对质量大小 来分配系统的输入输出。而在副产品再利用过程中, 减少 的废物处理量属于稻谷加工子系统, 减少的原材料投入则 属于副产品加工子系统。各子系统间的环境负荷选用对等 分配法 [10]进行分配, 即输入的原料稻谷和塑料编织袋在 其生产过程中产生的环境负荷和产品大米最终作为废物
处理造成的环境负荷由稻谷、塑料编织袋生产和废物处理 系统各承担 $50 \%$; 副产品稻壳、米糠、碎米粒的再循环过 程所造成的环境负荷由大米加工系统和副产品再循环系 统各负担 $50 \%$ 。

\subsection{4 . 清单结果}

根据软件模型换算及物流、能流和排放的分配，得到 大米加工系统资源能源消耗清单和环境释放清单, 部分清 单结果见表2和表3。

表2 部分资源能源消耗清单。

\begin{tabular}{lll}
\hline 类别 & 元素/物质 & 数量 $(\mathbf{k g})$ \\
\hline & 原油 & $3.29 \times 10^{6}$ \\
非可再生能源 & 硬煤 & $1.17 \times 10^{6}$ \\
& 褐煤 & $1.4 \times 10^{5}$ \\
& 天然气 & $7.36 \times 10^{5}$ \\
非可再生元素 & 铁 & $9.25 \times 10^{3}$ \\
& 铜 & $1.46 \times 10^{2}$ \\
& 水 & $9.31 \times 10^{10}$ \\
可再生资源 & 气 & $2.5 \times 10^{7}$ \\
& 二氧化碳 & $-1.6 \times 10^{8}$ \\
非可再生资源 & 惰性岩 & $6.81 \times 10^{6}$ \\
& 石灰岩 & $1.8 \times 10^{6}$ \\
\hline
\end{tabular}


表3 部分环境释放清单。

\begin{tabular}{lll}
\hline 类别 & 元素/物质 & 数量 \\
\hline & $\mathrm{CO}$ & $1.4 \times 10^{6}$ \\
& 镍 & 16.98 \\
& 氨 & $1.82 \times 10^{5}$ \\
& $\mathrm{CO}_{2}$ & $5.59 \times 10^{7}$ \\
大气排放 & $\mathrm{N}_{2} \mathrm{O}$ & $7.13 \times 10^{5}$ \\
& 甲烷 & $5.65 \times 10^{6}$ \\
& $\mathrm{PM}_{2.5}$ & $1.06 \times 10^{4}$ \\
& 农药类 & $3.88 \times 10^{4}$ \\
& 放射性物质 & $5.29 \times 10^{-6}$ \\
& $\mathrm{COD}$ & $5.73 \times 10^{3}$ \\
& 氯化物 & $7.28 \times 10^{5}$ \\
淡水排放 & 氮 & $3.67 \times 10^{5}$ \\
& 农药类 & $1.35 \times 10^{8}$ \\
\hline
\end{tabular}

\section{3. 影响评价}

\section{3.1. 分类}

本文将清单数据分为全球变暖潜能 (GWP)、酸化潜 能 (AP) 、富营养化潜能 (EP)、化石燃料消耗潜能(ADP fossil)、淡水生态毒性潜能(FAETP)、人类毒性潜能 (HTP)、
光化学氧化潜能 (POCP) 、陆地生态毒性潜能 (TETP) 8类。并应用GaBi6.0软件选取CML2001-Jan.2016模型进行 特征化计算, 标准化基准值也采用软件CML2001-Jan.2016 模型中的全球基准值。各影响类别的主要影响物质、基准 单位和标准化基准值见表4。

表4 环境影响分类及标准化基值。

\begin{tabular}{llll}
\hline 影响类型 & 主要元素/物质 & 基准单位 & 标准化基值 \\
\hline $\mathrm{GWP}$ & $\mathrm{CO}_{2} 、 \mathrm{CH}_{4} 、 \mathrm{~N}_{2} \mathrm{O} 、 \mathrm{HFCs}$ & $\mathrm{kg} \mathrm{CO}_{2}$-Equiv & $4.22 \times 10^{13}$ \\
$\mathrm{AP}$ & 氨、NOx、SO & $2.39 \times 10^{11}$ \\
$\mathrm{EP}$ & $\mathrm{COD} 、 \mathrm{~N} 、 \mathrm{P}$ SO -Equiv & $1.58 \times 10^{11}$ \\
$\mathrm{ADP}$ fossil & 硬煤、原油、褐煤、天然气、泥煤 & $\mathrm{kg}$ Phosphate-Equiv & $3.8 \times 10^{14}$ \\
FAETP & 农药、Co、 $、$ & $\mathrm{MJ}$ & $2.36 \times 10^{12}$ \\
$\mathrm{HTP}$ & $\mathrm{Cr}$ 二㜯英、2,3,7,8-TCDD、PAHs & $\mathrm{kg}$ DCB-Equiv & $2.58 \times 10^{12}$ \\
POCP & $\mathrm{CH}_{4} 、 \mathrm{NMVOC、NOx}$ & $\mathrm{kg}$ DCB-Equiv & $3.68 \times 10^{10}$ \\
$\mathrm{TETP}$ & kg Ethene-Equiv & $1.09 \times 10^{12}$ \\
\hline
\end{tabular}

\section{3.2. 特征化}

特征化可以将每一个影响类目中的不同物质转化和汇总成为统一的单元，并将其对环境的影响贡献程度量化。经 软件计算，大米加工系统的特征化总值见表5，各环节对不同影响类型的相对贡献度见图3-10。

表5 大米加工系统生命周期清单特征化结果。

\begin{tabular}{lllll}
\hline 影响类型 & GWP $\left(\mathbf{k g C O}_{2}\right.$-Equiv $)$ & AP $\left(\right.$ kgSO $_{2}$-Equiv $)$ & EP $($ kgPhosphate-Equiv $)$ & ADP fossil $(\mathrm{MJ})$ \\
\hline 总值 & $4.72 \times 10^{8}$ & $7.89 \times 10^{5}$ & $2.3 \times 10^{5}$ & $2.14 \times 10^{8}$ \\
影响类型 & FAETP (kgDCB-Equiv) & HTP $($ kgDCB-Equiv) & POCP (kg Ethene-Equiv) & TETP (kgDCB-Equiv) \\
总值 & $1.4 \times 10^{7}$ & $2.1 \times 10^{6}$ & $1.29 \times 10^{5}$ & $2.09 \times 10^{4}$ \\
\hline
\end{tabular}

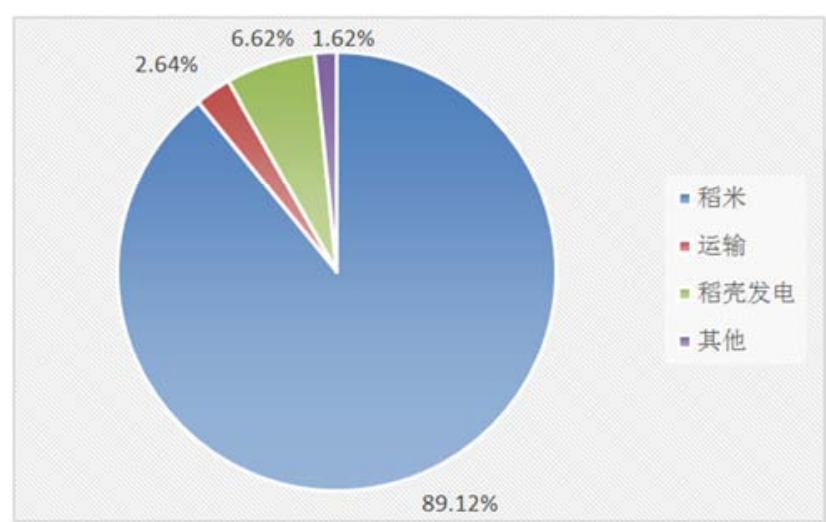

图3 全球变暖潜能。

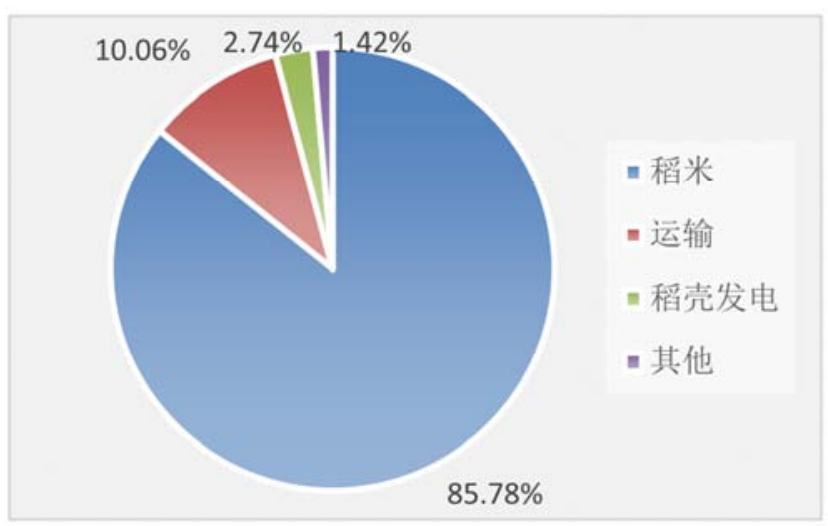

图4 酸化潜能。 


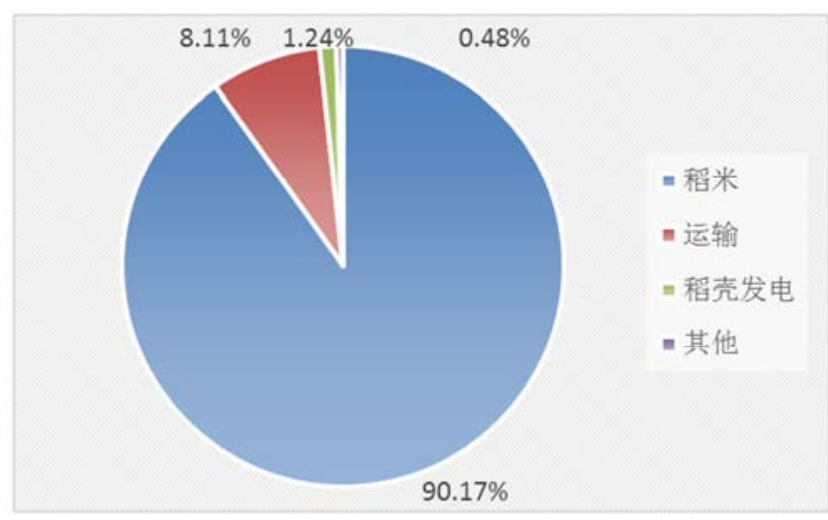

图5 富营养化潜能。

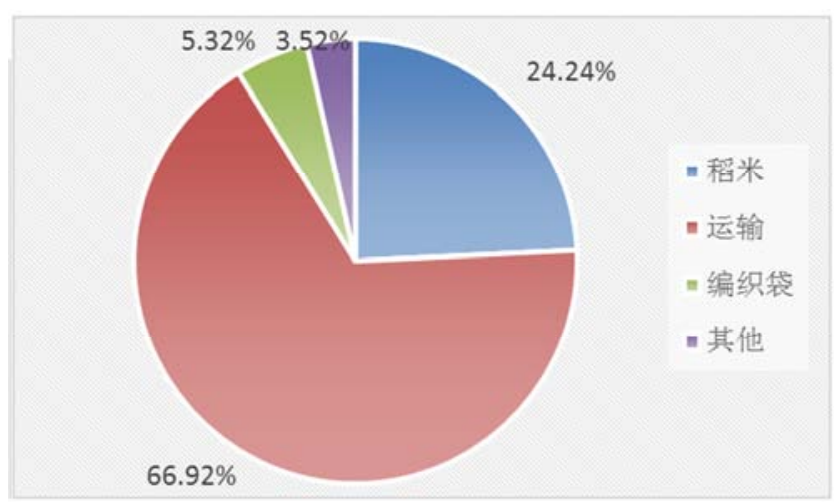

图6 化石燃料消耗潜能。

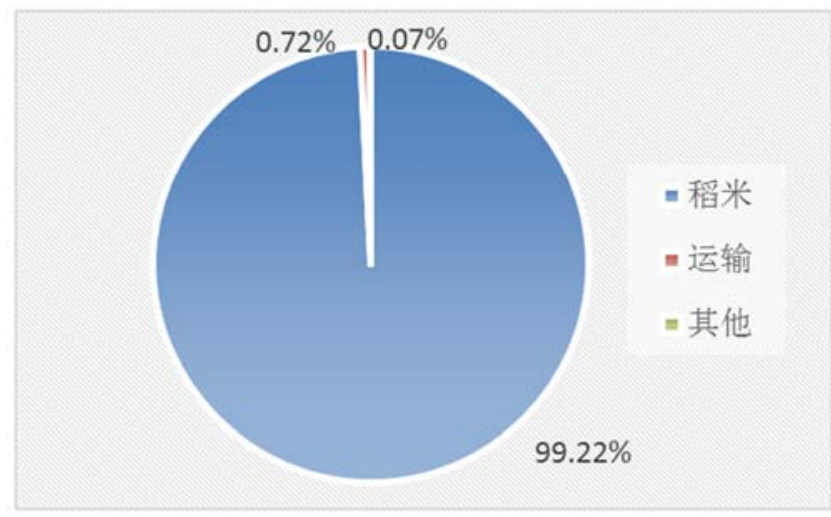

图7 淡水生态毒性潜能。

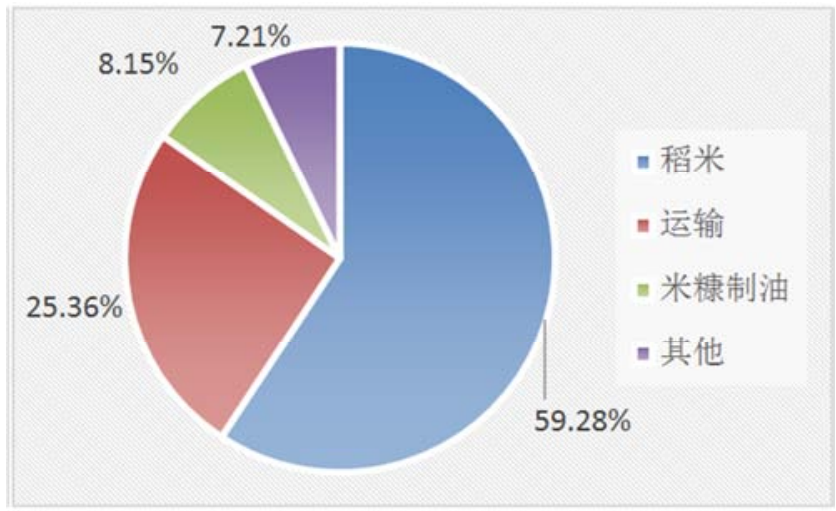

图8 人类毒性潜能。

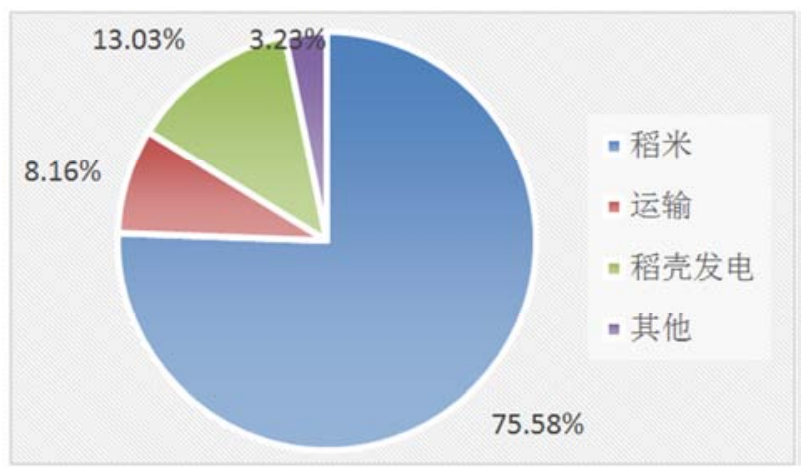

图9 光化学氧化潜能。

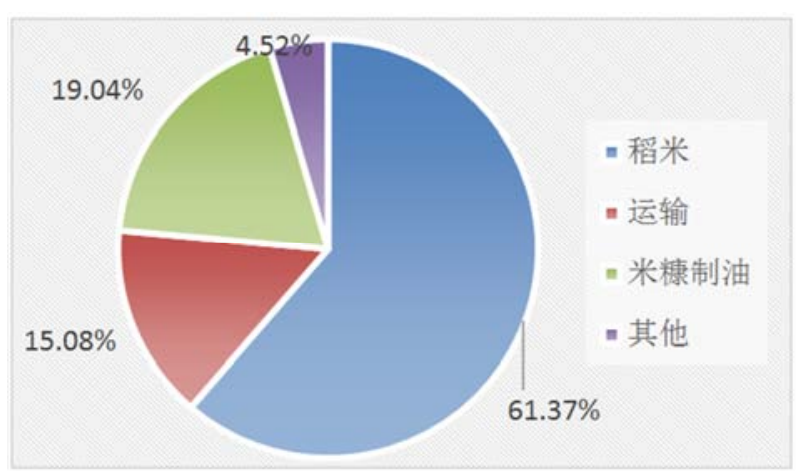

图10 陆地生态毒性潜能。

由图可知, 在大米加工系统中, 原料稻谷获取环节 (即 稻谷种植阶段) 产生的环境影响负荷最大, 其次是运输环 节。除化石燃料消耗潜能外, 稻谷种植环节对其它7种环境 影响类型的贡献率均大于其它环节贡献率的总和, 对全球 变暖潜能、酸化潜能、富营养化潜能和淡水生态毒性潜能 的贡献率分别达到了 $89.12 \% 、 85.78 \%$ 、 $90.17 \%$ 和 $99.22 \%$ 。 稻谷种植阶段，化肥生产过程 $\mathrm{CO}_{2}$ 的排放和淹水期 $\mathrm{CH}_{4}$ 的排 放是影响全球变暖的主要因素, 氮肥施用造成的 $\mathrm{NH}_{3}$ 排放 是影响酸化和富营养化的主要因素, 淹水期 $\mathrm{CH}_{4}$ 和 $\mathrm{NO}_{\mathrm{x}}$ 的排 放是影响光化学氧化的主要因素, 农药的使用是影响淡水 毒性、人类毒性和陆地毒性的主要因素 $[11,12]$ 。运输环节 对化石燃料消耗潜能和人类毒性潜能的贡献率分别为 $66.92 \%$ 和 $25.36 \%$ 。这是因为长距离的运输消耗了大量的柴 油, 柴油生产过程会消耗大量原油、天然气等非可再生资 源, 释放重金属、氮氧化物等有害物质; 运输尾气也含有 大量氮氧化物。

稻壳发电环节对光化学氧化潜能的贡献率为 $13.03 \%$, 这主要是因为稻壳燃烧过程中产生 $\mathrm{CO}$ 、和碳氢化合物。 编织袋对化石燃料消耗潜能的贡献率为 $5.32 \%$, 来自于编 织袋原材料聚乙烯和聚丙烯生产过程的消耗。米糠制油环 节对人类毒性潜能和陆地生态毒性潜能的贡献率分别为 $8.15 \%$ 和 $19.04 \%$, 主要贡献者是生产用电, 燃煤发电过程 产生的环境影响。而稻谷加工环节消耗的电能约是米糠制 油环节的10倍, 但该环节对每种环境影响类型的贡献都非 常小, 这主要是因为稻谷加工用电来自于稻壳发电而非燃 煤发电。此外, 由于系统内碎米产量相对较小且碎米加工 工艺简单, 该环节对每种环境影响类型的贡献基本都是最 小的。 


\section{3. 3. 标准化}

标准化可以将各类环境影响转化为一个相对于总的 环境影响的无量纲的值, 使不同类型的环境影响之间可以
相互比较。将特征化结果与CML2001-Jan.2016模型中的全 球基准值相除进行标准化计算, 具体标准化结果见表6, 其结果对比见图11。

表6 大米加工系统生命周期清单标准化结果。

\begin{tabular}{|c|c|c|c|c|c|c|c|c|}
\hline 影响类型 & 稻谷生产 & 编织袋生产 & 稻谷加工 & 稻壳发电 & 碎米加工 & 米糠制油 & 运输 & 总量 \\
\hline GWP & $9.96 \times 10^{-6}$ & $7.43 \times 10^{-9}$ & $1.6 \times 10^{-7}$ & $7.39 \times 10^{-7}$ & $1.88 \times 10^{-9}$ & $1.22 \times 10^{-8}$ & $2.95 \times 10^{-7}$ & $1.12 \times 10^{-5}$ \\
\hline $\mathrm{AP}$ & $2.83 \times 10^{-6}$ & $3.05 \times 10^{-9}$ & $1.95 \times 10^{-8}$ & $9.05 \times 10^{-8}$ & $1.4 \times 10^{-9}$ & $2.3 \times 10^{-8}$ & $3.32 \times 10^{-7}$ & $3.3 \times 10^{-6}$ \\
\hline EP & $1.31 \times 10^{-6}$ & $3.91 \times 10^{-10}$ & $3.89 \times 10^{-9}$ & $1.8 \times 10^{-8}$ & $1.5 \times 10^{-10}$ & $2.54 \times 10^{-9}$ & $1.18 \times 10^{-7}$ & $1.46 \times 10^{-6}$ \\
\hline ADP fossil & $1.37 \times 10^{-7}$ & $3 \times 10^{-8}$ & $4.4 \times 10^{-10}$ & $2.04 \times 10^{-9}$ & $2.09 \times 10^{-9}$ & $1.53 \times 10^{-8}$ & $3.77 \times 10^{-7}$ & $5.63 \times 10^{-7}$ \\
\hline FAETP & $5.9 \times 10^{-6}$ & $1.1 \times 10^{-9}$ & $8.38 \times 10^{-11}$ & $3.88 \times 10^{-10}$ & $3.28 \times 10^{-10}$ & $2.07 \times 10^{-9}$ & $4.27 \times 10^{-8}$ & $5.95 \times 10^{-6}$ \\
\hline HTP & $4.83 \times 10^{-7}$ & $3.92 \times 10^{-9}$ & $1.01 \times 10^{-8}$ & $3.37 \times 10^{-8}$ & $1.09 \times 10^{-8}$ & $6.64 \times 10^{-8}$ & $2.06 \times 10^{-7}$ & $8.14 \times 10^{-7}$ \\
\hline TETP & $1.18 \times 10^{-8}$ & $8.69 \times 10^{-11}$ & $2.97 \times 10^{-11}$ & $1.38 \times 10^{-10}$ & $6.11 \times 10^{-10}$ & $3.65 \times 10^{-9}$ & $2.89 \times 10^{-7}$ & $1.92 \times 10^{-8}$ \\
\hline
\end{tabular}

可以看出，大米生产系统对环境损害最大的影响类型是全球变暖，其损害为 $1.12 \times 10^{-5}$ 。其次是淡水生态毒性、光 化学氧化和富营养化, 其损害分别为 $5.95 \times 10^{-6} 、 3.51 \times 10^{-6}$ 和 $3.3 \times 10^{-6}$ 。这说明水稻种植淹水期以及化肥农药的施用是对 环境影响损害的最大贡献者。

\section{3. 4. 权重}

通过查阅文献, 以对全球的环境影响为尺度, 确定 8 种环境影响类型两两之间的重要性关系, 利用层次分析法计算 各影响类型的权重, 并对标准化结果加权计算, 各影响类型权重及加权总值见表 7。本次研究系统总的环境影响为 $4.35 \times 10^{-6}$, 全球变暖是对环境产生影响的主要因素, 占总环境影响的 $66.58 \%$; 其次是淡水生态毒性, 占总环境影响的 $18.68 \%$ 。

表7 八种影响类型的权重和加权结果。

\begin{tabular}{|c|c|c|c|c|c|c|c|c|}
\hline 影响类型 & GWP & $\mathbf{A P}$ & EP & ADP fossil & FAETP & HTP & РOCP & TETP \\
\hline 权重 & 0.259 & 0.0431 & 0.0636 & 0.021 & 0.136 & 0.353 & 0.0296 & 0.0938 \\
\hline 总值 & $2.89 \times 10^{-6}$ & $1.42 \times 10^{-7}$ & $9.27 \times 10^{-8}$ & $1.19 \times 10^{-8}$ & $8.12 \times 10^{-7}$ & $2.88 \times 10^{-7}$ & $1.04 \times 10^{-7}$ & $1.8 \times 10^{-9}$ \\
\hline
\end{tabular}

\section{4. 敏感性分析}

结合影响评价结果, 本文选取了对总环境影响负荷贡 献超过 $5 \%$ 的 3 个环节, 分别是稻谷种植、稻壳发电和运输, 并以相关输入数据为变量进行敏感性分析。对原料稻谷输 入、发电环节稻壳输入和运输距离 3 个因子分别改变 $10 \%$, 计算得出全生命周期环境影响负荷的变化比例, 结果见表 8。可以看出, 原料稻谷输入量的改变对全生命周期环境 影响负荷所造成的影响最大。

表8 敏感性分析结果。

\begin{tabular}{ll}
\hline 变量 & 环境影响负荷变化率 \\
\hline 稻谷 & $8.84 \%$ \\
稻壳 & $0.51 \%$ \\
运输距离 & $0.44 \%$ \\
\hline
\end{tabular}

\section{4. 结论与建议}

从大米加工系统的全生命周期看, 全球变暖和淡水生 态毒性是主要的环境影响因素, 年产19万吨大米全球变暖 潜值 ( $\mathrm{CO}_{2}$-Equiv) 和淡水生态毒性潜值（DCB-Equiv）分 别为 $4.72 \times 10^{8} \mathrm{~kg}$ 和 $1.4 \times 10^{7} \mathrm{~kg}$ 。

从生命周期各环节来看, 稻谷种植环节是大米加工系 统的高污染环节, 占总环境影响的 $88.4 \%$ 。改进水稻灌溉方
式（如地下滴灌[13]），控制化肥和农药的用量可有效降低 稻谷种植过程的环境影响。运输环节是化石燃料消耗潜能 的主要影响因素, 在人类毒性潜能中也占有较大比重。运 输同重量货物相同距离公路运输产生的环境影响是铁路运 输的几倍, 因此, 选择合适的运输方式、制定合理的运输 路线是减缓该环节环境影响的关键。稻谷加工和各副产品 加工环节中, 稻壳发电对环境的影响最大。提高稻壳的燃 烧效率, 优化烟风系统（如利用烟气余热等）, 改进烟气 脱硫脱硝工艺[14], 可有效降低稻壳发电对环境的影响。

此外, 尽管本系统塑料编织袋的消耗量相对很小, 其 环境影响却占化石燃料消耗潜能的 $5.32 \%$ 。这是因为本次 研究将原油作为塑料编织袋原料聚乙烯、聚丙烯的生产原 料进行测算, 以可再生能源 (如甘蔗) 代替原油可减少化 石燃料的消耗和碳排放[13]。

\section{参考文献}

[1] 崔雯雯. 基于图像处理的大米品质检测系统研究 [D]. 吉林: 吉林大学, 2015。

[2] 中国统计出版社, 中国统计年鉴[M], 2016。 
[3] 于瑾泉, 张芸, 张树深, 等. 啤酒的生命周期评价 [J]. 环 境科学学报, 2015, (04):1217-1223。

[4] Kwofie E. M, Ngadi M. A. comparative lifecycle assessment of rural parboiling system and an integrated steaming and drying system fired with rice husk[J]. Journal of Cleaner Production, 2017, 140: 622-630.

[5] Roy P, Ijiri T, Nei D, et al. Life cycle inventory (LCI) of different forms of rice consumed in households in Japan[J]. Journal of Food Engineering, 2009, 91(1): 49-55.

[6] Frischknecht R, Fantke P, et al. Global guidance on environmental life cycle impact assessment indicators: progress and case study[J]. International Journal of Life Cycle Assessment, 2016, 21(3): 429-442.

[7] 林涛. 大米加工节能降耗技术的研究 [J]. 粮食与食品工业, 2015, (04):42-43+49。

[8] 余红. 可再生能源发电对PM_(10)和PM_(2.5)减排的贡献核 算 [J]. 环境工程技术学报, 2014, (04):321-325。
[9] 于跃龙, 于丽伟, 吕淑华. 利用碎米加工大米粉的生产工 艺研究 $[J]$. 黑龙江粮油科技, 1998, (01):54-55。

[10] 邓南圣, 王小兵. 生命周期评价[M]. 北京: 化学工业出版 社, 2013:107-108。

[11] 梁龙, 陈源泉, 高旺盛. 两种水稻生产方式的生命周期环 境影响评价[J]. 农业环境科学学报, 2009, (09):1992-1996。

[12] 王明新, 夏训峰, 刘建国, 柴育红, 雷春生. 太湖地区高 产水稻生命周期评价 [J]. 农业环境科学学报, 2009, (02):420-424。

[13] Coltro L, Marton L. F. M, Pilecco F. P, et al. Environmental profile of rice production in Southern Brazil: A. comparison between irrigated and subsurface drip irrigated cropping systems [J]. Journal of Cleaner Production, 2016.

[14] 朱金伟, 张凡, 王洪昌, 王凡, 束韫. 燃煤烟气脱硫脱硝 技术的发展趋势[J]. 环境工程技术学报, 2015, (03):200-204。 